\title{
Improving the quality of patient handover on a surgical ward
}

\author{
Alison Bradley
}

NHS Tayside

\begin{abstract}
The European Working Time Directive means safe patient hand over is imperative. It is the responsibility of every doctor and an issue of patient safety and clinical governance [1]. The aims of this project were to improve the quality of patient handover between combined assessment unit (CAU) and surgical ward FY1 doctors.
\end{abstract}

The Royal College of Surgeons England (RCSEng) guidelines on surgical patient handover [1] were used as the standard. Data was collected throughout November 2013. A handover tool was then introduced and attached to the front of patient notes when a patient was transferred from CAU to the surgical ward. The doctor handing over the patient and the ward doctor receiving the handover signed this document. Policy was also changed so that handover should take place once the patient had received senior review on the CAU and was deemed appropriate for transfer to the surgical ward. Data from the handover tool was collated and checked against the list of surgical admission for February 2014.

The number of patients handed over improved from $15 \%$ to $45 \%$. The quality of patient handover also improved. 0 patient handovers in November 2013 included all of the information recommended by the RCSEng guidelines. 100\% of the patient handovers in February 2014 contained all the recommended information.

Introduction of a handover tool and formalisation of timing of patient handover helped to improve quality and number of patients being handed over. Further work needs to be done to improve safe handover of surgical patients, particularly out of hours.

\section{Problem}

Perth Royal Infirmary is a busy district general hospital in Scotland. Emergency surgical admissions are admitted to a Combined Assessment Unit (CAU) Monday to 6pm Friday. They are clerked by a FY1 doctor and then have a senior review by a registrar and/or consultant surgeon before either being deemed fit to be transferred to the surgical ward, admitted to HDU, or transferred to another hospital. The same on-call registrar is responsible for the patients admitted both in CAU and when they are transferred within the hospital to another ward.

After senior review the patients are transferred sporadically depending on bed availability on the surgical ward. Often the FY1 on CAU is not informed that the patient has been transferred. This impedes a time appropriate handover between CAU and surgical ward FY1s, and potentiates delays in ordering, chasing, and acting quickly on investigations and their results, particularly as the on-call registrar is often scrubbed in theatre. Clearly this has patient safety implications.

A formal handover of patients occurs at 9pm every night and is attended by the day FY1, on-call registrar, and hospital at night team, which includes an FY2 or GPST1-2, senior nursing staff, and support workers. There is no registrar on-call at night. A consultant surgeon is on-call at home. There is no formal patient handover from the night team back to the day team in the morning as the night surgical doctor is expected to attend the orthopaedic trauma meeting which takes place at the same time the surgical FY1s start their shift. Information from the night team is usually communicated via a handover sheet.

\section{Background}

European Working Time Directive and subsequent change in shift patterns means that good quality patient handover is imperative to patient safety [1]. Recent studies have highlighted the potentially disastrous compromise to patient safety caused by poor quality patient handover [2,3].

Safe patient handover is the responsibility of every doctor and must be taught and developed [1]. Whilst there is no definitive way in which this can be achieved, clear guidelines on the safe handover of surgical patients exist [1]. Juxtapose this with the fact that junior doctors tend to be heavily involved in handovers from an early stage in their career, and it is clear that they must be trained and empowered to perform this skill well so that patient safety can be improved.

A plethora of approaches have been tried in projects to improve patient handover. Most involve the introduction of a handover tool to succinctly summarise information. Din et al. [4] created a tool which used a traffic light system to help staff appropriately prioritise patients requiring weekend review. This was shown to improve quality of, and confidence in, weekend handover of surgical patients, as well as improve efficiency of weekend ward rounds. However, it would be difficult to utilise a traffic light approach in improving handover of patients between $\mathrm{CAU}$ and the surgical ward 
due to the unpredictable nature of acute admissions in relation to prioritising workload.

Din et al. [4] did corroborate findings from other studies that formalising handover through introduction of a handover tool improved quality of handover [5,6,7]. Ashton [5] incorporated SBAR (Situation, Background, Assessment and Recommendations) in their handover tool. This study did however focus on weekend handover of medical patients and information is not yet available on how successfully this tool was when introduced into surgical departments. SBAR approach alone would not necessarily guarantee that all information recommended in the RCSEng guidelines for handover of surgical patients would be included [1]. Other studies focused on introducing a formalised electronic handover tool $[6,7]$. Whilst it is currently unfeasible to introduce an electronic handover format in our current setting, lessons could be drawn from the value found not only in introducing a formalised tool but also in attaching accountability to the information handed over [6].

\section{Baseline measurement}

Before initiating this project all surgical admissions during November 2013 were audited. Surgical ward FY1s completed an audit sheet each time they encountered a new patient on the surgical ward. At the end of each shift the registrar checked that an audit sheet had been completed for each patient moved from CAU to the surgical ward that day.

Outcomes measured included: were patients handed over, how FY1s became aware of new patients on the ward, contents of handover if any took place, and times of patient admission and transfer from CAU to surgical ward. Additionally information including SEWS score at time of admission and at time of transfer were also gathered from patient notes.

68 cases were audited. 9 were excluded as a full data set was not recorded giving a total of 59 cases. $15 \%$ of patients were handed over to the FY1. FY1s reported that they first became aware of new admissions by encountering them during a ward round in $48 \%$ of cases. $17 \%$ of new admissions were discovered by the FY1s when they were asked to do a job in relation to the patient. In $10 \%$ of new admission FY1s became aware of the new admission when they were asked to review them, and in $9 \%$ of admissions the FY1 noticed a new name on the ward board.

Of the new admissions who had an increase in their SEWs score between CAU and ward 1, 40\% were handed over to the surgical ward $\mathrm{FY} 1$ but $60 \%$ were not.

Of those patients who were handed over 0 included all the recommended minimal data set from the RCSEng guidelines.

\section{Design}

When it came to considering the cause of the problem it became clear that patients were being moved from the CAU to the surgical ward without effective communication between CAU nurses and the CAU FY1. Also, FY1 doctors were not aware of guidelines concerning the safe handover of surgical patients. Knowledge deficit and better communication pathways therefore needed to be addressed. Creation of a handover tool and introduction of a policy concerning the timing of patient handover were therefore introduced (Figure 1).

\section{Strategy}

PDSA Cycle 1: Consultants were approached to find out what was already known about the quality of handover of acute surgical admission between the CAU and surgical ward FY1s. As predicted only anecdotal evidence existed which suggested improvement should be made. It was agreed that an audit of current practice be undertaken.

PDSA Cycle 2: Findings from the initial audit were presented at the hospital Clinical Effectiveness Meeting. As predicted this corroborated anecdotal evidence that handover of acute surgical admissions from CAU to the surgical ward could be improved. A series of sessions were agreed upon to discuss how practice could be improved.

PDSA Cycle 3: A feedback session was arranged with consultants and registrars. It was decided that a policy should be introduced whereby patient handover should take place between CAU and surgical ward FY1s as soon as the patient had had a senior review in CAU and was deemed appropriate to transfer to the surgical ward. It was felt that formalising timing of handover in this way would better facilitate handover.

PDSA Cycle 4: In addition to this top-down approach, sessions were also held with FY1 doctors working in the surgical department in both CAU and the surgical ward. These sessions included teaching on guidelines for information to be included in surgical patient handovers. Whilst the FY1s agreed with the consultant led change in policy, they also highlighted that time pressure made it difficult to remember the RCSEng minimal data set to be included in handover of surgical patients [1]. Also it was decided that structuring the roles of FY1s on the surgical ward so that one FY1 was named as being responsible for new admissions during their shift, would simplify and improve communication processes between CAU and the ward.

PDSA Cycle 5: The changes outlined in cycle 4 were also agreed at consultant level.

PDSA Cycle 6: A handover tool, based on the RCSEng guidelines [1], was created and refined following qualitative feedback from Consultants and FY1 doctors. Signatures from the CAU FY1 giving, and surgical FY1 receiving, handover were added as it was felt that this would support the policy of having one ward FY1 dealing with new admissions, hence simplifying communication processes between the two wards.

PDSA Cycle 7: Teaching sessions took place on how and when to use the new handover tool. 
PDSA Cycle 8: The handover tool and new handover policy were piloted for a 1 week period. No negative feedback was received.

PDSA Cycle 9: The handover tool and policy were implemented from 1st-25th March and their impact re-audited.

\section{Results}

Post-intervention measurements were carried out on all patients admitted between 08:00 and 22:00 from 1st to 25th February 2014. This gave a total of 56 patients. The number of patients handed over between CAU and surgical ward FY1s increased from 15\% to $45 \%$. The quality of patient handover using the handover tool increased from $0 \%$ of handovers including the recommended minimal data set to $100 \%$ of those handed over including all information in the recommended data set [1].

See supplementary file: ds3114.doc - "Figure 1: Handover tool"

\section{Lessons and limitations}

This project shows that good quality patient handover is a skill that can be taught and developed. This process is facilitated by also improving communication systems. However, it is limited by small numbers and there is a potential for bias as the doctors involved knew that the audit was taking place.

The project focuses on, and achieved, improvement in the quality of handover but does not look at quality of care initiated in CAU, or how a multi-disciplinary approach could further improve quality and frequency of patient handover. Also, issues concerning handover at weekends/ out-of-hours are not dealt with. These are intended areas of focus for future projects.

\section{Conclusion}

Introduction of a handover tool, supported by a change in policy regarding handover of surgical admissions, improved the quality of patient handover. This shows that good patient handover is a skill that can, and should, be taught and developed. Some improvement was shown in the number of patients handed over between CAU and the ward but clearly further improvement is required. Future projects will seek to address this by looking at multi-disciplinary handover tools and training.

\section{References}

1. Royal college Surgeons England, Safe handover: guidance from the Working Time Directive working party, RCSEng, March 2007.

2. Freemantle N, Richardson M, Wood J, et al. (2012) Weekend hospitalization and additional risk of death: An analysis of inpatient data. J R Soc Med. Feb;105(2):74-84.

3. The Royal College of Surgeons of England (2011). The Higher Risk General Surgical Patient: towards improved care for a forgotten group, RCSE, London. Available at: http://www.rcseng.ac.uk/publications/docs/higher-risksurgical-patient

4. Din N, Ghaderi S, O'Connell R, et al. (2012) Strengthening surgical handover: Developing and evaluating the effectiveness of a handover tool to improve patient safety. BMJ Quality Improvement Reports. 1,No. 1u492.w164 doi: 10.1136/bmjquality.u492.w164

5. Ashton C. (2013). Improving weekend patient handover. BMJ Quality Improvement Reports 2013;u201303.w827doi:10.1136/bmjquality.u201303.w827

6. Till A, Sall H, Wilkinson J. (2014). Safe Handover : Safe Patients - The Electronic Handover System. BMJ Quality Improvement Reports 2014; u202926.w1359doi:10.1136/bmjquality.u202926.w1359

7. Mehra A, Henein C. (2014). Improving hospital weekend handover: a user-centered, standardised approach. BMJ Quality Improvement Reports 2014; u202861.w1655 doi:10.1136/bmjquality.u202861.w1655

\section{Declaration of interests}

Nothing to declare

\section{Acknowledgements}

Perth Royal Infirmary Surgical Department 